\title{
An acute transfusion reaction
}

\author{
Authors: Clare Webb, ${ }^{A}$ Alan Norris ${ }^{B}$ and Katie Hands ${ }^{C}$
}

\begin{abstract}
We present the case of a 67-year-old man who suffered an acute anaphylactic reaction during red cell transfusion due to the presence of anti-IgA antibodies. The incidence and clinical relevance of anti-IgA antibodies in IgA deficiency is reviewed, and the wider investigation and management of acute transfusion reactions is also discussed. This case highlights the need to consider the potential risks of blood component transfusion against the purported benefit.
\end{abstract}

KEYWORDS: Transfusion reaction, IgA deficiency, anaphylaxis

\section{Case presentation}

A previously fit and well 67-year-old man presented to his GP with an 8-week history of shortness of breath and chest tightness on exertion. Initial investigations revealed a macrocytic anaemia with haemoglobin $(\mathrm{Hb}) 88 \mathrm{~g} / \mathrm{L}$ and mean corpuscular volume (MCV) $140 \mathrm{fL}$. Vitamin B12 levels were low at $110 \mathrm{ng} / \mathrm{L}$. A working diagnosis of pernicious anaemia was reached. Further blood samples were sent, aiming to confirm a diagnosis of pernicious anaemia; anti-intrinsic factor, anti-gastric parietal cell antibodies, and to assess for malabsorption; anti-tissue transglutaminase and anti-endomysial antbiodies, as well as total immunoglobulin levels. A red cell transfusion was arranged in the community hospital on account of the exertional cardiac symptoms.

Ten minutes into the transfusion, the patient started to feel unwell and sweaty. He was afebrile with no tachycardia or change in his blood pressure. He was reviewed by medical staff and the rate of the red cell transfusion was slowed. The patient felt progressively more unwell and sweaty and had a sense of impending doom. Sixty minutes into the transfusion he spiked a fever of $38.2^{\circ} \mathrm{C}$, his systolic blood pressure dropped from 140 to $90 \mathrm{mmHg}$ and he had a widespread urticarial rash. He had no lip or throat swelling and no wheeze.

\section{Diagnosis}

Types of acute transfusion reaction are summarised in Box 1. Initial management of an acute transfusion reaction is not

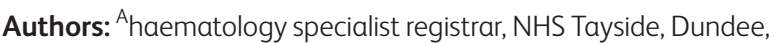
UK; ${ }^{B}$ associate specialist in blood transfusion, Scottish National Blood

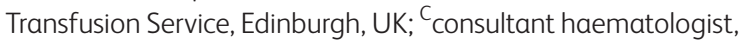
Scottish National Blood Transfusion Service, Edinburgh, UK
Box 1. Types of acute transfusion reaction

Non-haemolytic
$>$ Bacterial contamination
$>$ Febrile non-haemolytic transfusion reaction
$>$ Allergic reaction
$>$ Anaphylactic reaction
$>$ Transfusion-related acute lung injury
$>$ Transfusion-associated circulatory overload
Haemolytic
$>$ ABO incompatibility (immediate intravascular red cell
destruction)
$>$ Other red cell incompatibility eg Rhesus, Kell (extravascular red
cell destruction)

dependent on classification as treatment cannot be delayed pending investigations. Rapid onset of shock, with lip or throat swelling, or urticarial rash, suggests anaphylaxis.

\section{Initial management}

The transfusion was stopped and prompt airway, breathing and circulatory assessment undertaken. The blood component bag and label were checked against the patient's details and no discrepancy found. He was immediately treated with IV saline, hydrocortisone and chlorpheniramine. He was transferred by blue light ambulance to the Emergency Department, where he received further treatment for anaphylaxis. Management of anaphylaxis, after immediate resuscitation, is the administration of intramuscular adrenaline. ${ }^{2}$ Intravenous hydrocortisone will shorten the duration of an anaphylactic reaction, and the evidence for intravenous chlorpheniramine is weaker, but this is also commonly administered. ${ }^{3}$

The patient was investigated for the acute transfusion reaction. Standard investigations, including full blood count, renal and liver function tests, and tests for haemolysis were performed. As the fever was sustained, the implicated unit was returned to the laboratory for repeat compatibility testing and the patient retested for red cell antibodies. The unit was sent for bacterial culture.

The blood transfusion service consultant on call was contacted. The transfusion reaction was reported to the Serious Hazards of Transfusion (SHOT) haemovigilance reporting scheme. A blood 
Table 1. Results of initial blood tests

\begin{tabular}{ll} 
Parameter & Value \\
Haemoglobin & $90 \mathrm{~g} / \mathrm{L}$ \\
WBC & $9.8 \times 10^{\wedge} 9 / \mathrm{L}$ \\
Platelets & $245 \times 10^{\wedge} 9 / \mathrm{L}$ \\
Mean cell volume & $130.4 \mathrm{fL}$ \\
Reticulocytes & $24 \times 109 / \mathrm{L}$ \\
Lactate & $1.2 \mathrm{mmol} / \mathrm{L}$ \\
Lactate dehydrogenase & $600 \mathrm{U} / \mathrm{L}$ \\
ALT & $41 \mathrm{U} / \mathrm{L}$ \\
Bilirubins & $13 \mu \mathrm{mol} / \mathrm{L}$ \\
Alkaline phosphatase & $113 \mathrm{U} / \mathrm{L}$ \\
Albumin & $37 \mathrm{~g} / \mathrm{L}$ \\
Creatinine & $68 \mu \mathrm{mol} / \mathrm{L}$ \\
Haptoglobin & $<0.08 \mathrm{~g} / \mathrm{L}$ \\
Direct Coombs testing: & \\
Anti - IgG & Negative \\
Anti - C3d & Negative \\
Blood group & $0 \mathrm{positive}$ \\
Antibody status & No red cell antibodies detected \\
\hline ALT = alanine transaminase; WBC $=$ white blood cell \\
\hline
\end{tabular}

bank search was performed so that any other components from the implicated donation could be withdrawn if necessary. ${ }^{2}$

Blood tests results are summarised in Table 1. Repeat compatibility testing confirmed no serological incompatibility between the patient and implicated donor red cells. The repeat antibody screen was negative. There was no bacterial growth on culture. Some biochemical markers of haemolysis were present, but not to the degree that would be expected in an acute haemolytic transfusion reaction. This is explained by the B12 deficiency, which causes friable cells prone to spontaneous lysis.

Patients who have experienced a severe allergic transfusion reaction should have IgA levels measured. ${ }^{2}$ In this case, recent immunology investigations already demonstrated isolated IgA deficiency with a level of $<0.0005 \mathrm{~g} / \mathrm{L}$. Subsequent investigation confirmed the presence of anti-IgA antibodies. See image 1. The diagnosis of anaphylaxis to IgA present in the donor red cell unit was reached.

\section{Case progression and outcome}

The patient was kept in the Emergency Department for observation for 6 hours, and went on to make a full recovery. His haemoglobin level and MCV later normalised after 4 weeks with parenteral vitamin B12 replacement.

\section{Discussion}

The incidence of anti-IgA mediated anaphylactic transfusion reactions is extremely low. The total annual incidence of severe anaphylactic or hypotensive acute transfusion reaction (excluding haemolytic reactions and transfusion related lung injury) was 86 out of 2.5 million blood transfusions in the UK in $2015 .{ }^{4}$ Only one

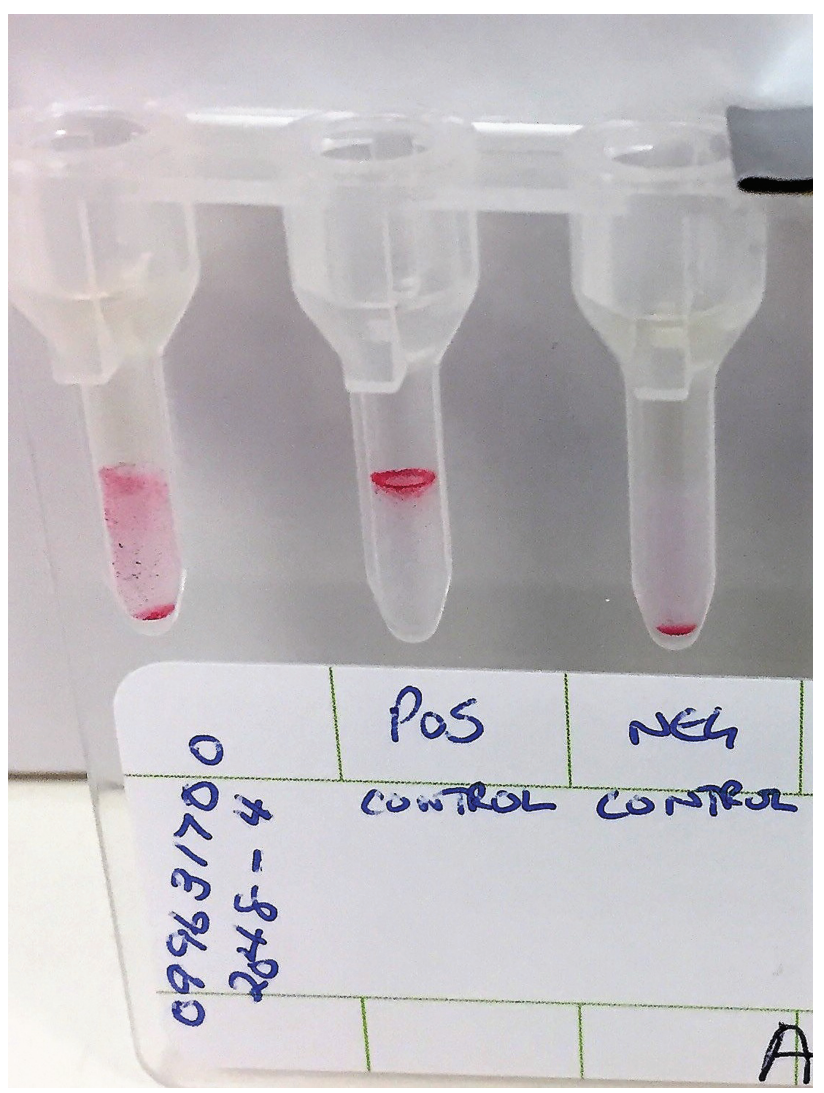

Fig 1. Testing for anti-IgA antibodies.

of these was found to be due to anti-IgA mediated anaphylaxis. Other estimates of incidence include 1 in 1.3 million blood components transfused. ${ }^{5}$

Isolated IgA deficiency, on the other hand, is very common with a prevalence of between 1 in 500 and 1 in 200. ${ }^{4,5}$ Although the rate of anti-IgA mediated anaphylaxis is higher in this group, it is still rare. The postulated mechanism for anaphylaxis involves sensitisation of the IgA-deficient patient via exposure to IgA, and anti-IgA mediated anaphylaxis on subsequent exposure. Our patient had never had a previous blood transfusion, and he could not recall having received any treatment which would have exposed him to IgA such as intravenous immunoglobulin or tetanus immunoglobulin. Many anti-IgA antibodies occur naturally; in one study, only 9 out of the 39 blood donors with detectable anti-IgA antibodies had a history of transfusion or pregnancy. ${ }^{6}$ It could be that sensitisation occurs in utero by maternal IgA crossing the placenta and entering the foetal circulation. Alternatively, human breast milk IgA, or bovine IgA, could be absorbed via the intestinal wall and cause sensitisation., 7.8

Patients with anti-IgA antibodies are at high risk of future anaphylaxis to blood products, because of IgA present in donor plasma. Red cells or platelets from IgA deficient donors are not routinely available. Red cells and platelets can be washed prior to issue to minimise donor plasma present in the component. This process requires planning and therefore washed components are predominantly used for elective transfusion or surgery. In a life-threatening emergency setting, if the risk of delay in waiting 
for washed cells outweighs the need for urgent transfusion, then standard cells should be used. Any transfusion must be in a setting where staff have been trained in anaphylaxis management and have access to adrenaline. Stocks of IgA deficient plasma are held at national centres and their issue requires discussion with a blood transfusion service consultant. $^{9}$

There are a small number of IgA deficient patients with antiIgA mediated anaphylaxis who require elective large volume transfusion (eg planned high risk surgery). Desensitisation is an option for this group. ${ }^{10,11}$

Patients with confirmed IgA deficiency, who have never previously been transfused, or who have received standard blood components with no reaction, should be transfused with standard components. ${ }^{9}$ The incidence of transfusion reaction, as discussed above, is extremely low.

\section{Key learning points}

$>$ Initial management of an acute transfusion reaction is independent of laboratory test results

> Management of an anaphylactic transfusion reaction includes prompt supportive measures and IM adrenaline

> Patients who suffer an anaphylactic transfusion reaction should have IgA levels measured

> In general blood transfusion is very safe. Risk of serious harm (including serious transfusion reaction or infection) in the UK is $<1$ in 15,000, and risk of death $<100,000^{4}$

$>$ Over 2.5 million units of blood product transfused annually in the UK. Minimisation of risk includes reviewing the indication for transfusion in every patient.

\section{Author contributions}

CW made the initial assessment of the patient in the Emergency Department. $\mathrm{KH}$ and $\mathrm{AN}$ organised further transfusion department testing and reporting. Initial writing of the case report was done by $\mathrm{CW}$ and part of the discussion and editing was done by $\mathrm{KH}$.

\section{Conflict of interest}

The authors declare no conflict of interest.

\section{Acknowledgements}

Written consent was obtained from the patient to publish the clinical details in this article.

David Ward and his team at the Red Cell Immunohaematology Laboratory, Sheffield Blood Centre, provided testing for anti-IgA antibodies and the photograph of this test.

\section{References}

1 Hoffbrand AV, Catovsky D, Tuddenham EGD. Postgraduate Haematology, 6th edn. John Wiley and Sons, 2010.

2 Tinegate $\mathrm{H}$, Birchall J, Gray A et al. Guideline on the investigation and management of acute transfusion reactions. $\mathrm{Br}$ ] Haematol 2012:159:143-53.

3 Serious Hazards of Transfusion (SHOT). SHOT bites No 5: Acute transfusion reactions - lessons for clinical staff. SHOT, 2015.

4 Serious Hazards of Transfusion (SHOT). Annual SHOT report. Serious Hazards of Transfusion SHOT, 2015.

5 Latiff AHA, Kerr MA. The clinical significance of immunoglobulin A deficiency. Ann Clin Biochem 2007:44:131-9.

6 Thibault $\mathrm{L}$ et al. Establishment of an immunoglobulin A-deficient blood donor registry with a simple in-house screening enzymelinked immunosorbent assay. Transfusion 2006:46:2115-21.

7 Laschinger CA and Naylor DH. IgA antibodies and transfusion reactions in Canada. Can Med Assoc J 1983:128:381-2.

8 Nadorp JH, Voss M, Buys WC et al. The significance of the presence of anti-IgA antibodies in individuals with an IgA deficiency. Eur ] Clin Invest 1973:3:317-23.

9 Latham T. Investigation and clinical management of suspected reactions to IgA. London: NHSBT, 2010. http://hospital.blood.co.uk/ clinical-guidelines/nhsbt-clinical-guidelines/

10 Kiani-Alikhan S, Yong PF, Grosse-Kreul D et al. Successful desensitization to immunoglobulin $\mathrm{A}$ in a case of transfusion-related anaphylaxis. Transfusion 2010;50:1897-901.

11 Salama A, Kardashi R, Arbach O. Long-term treatment and transfusion of normal blood components following tolerance induction in patients with anti-IgA anaphylactic reactions. Transfus Med Hemotherapy 2014;41:381-7.

Address for correspondence: Dr Clare Webb, Haematology Department, Ninewells Hospital and Medical School, Dundee DD1 9SY, UK.

Email: clare.webb5@nhs.net 